\title{
Comprehensive staff evaluation
}

\author{
Khalida Ahaverdiieva ${ }^{1, *}$, \\ ${ }^{1}$ Simon Kuznets Kharkiv National University of Economics, Department of Economics and social \\ Sciences, avenue Nauki, 9-a, 61166, Kharkiv, Ukraine
}

\begin{abstract}
Evaluation is indispensable for the staff management system of any company, as according to evaluation data any reasonable managerial decisions can be made in the field of staff rotation, training, establishment of salary amount, etc. The evaluation results constitute an important factor of performance motivation and positive change in approaches towards professional experience. Due to staff evaluation the efficient company can be established that ensures better conditions for development and growth of the employees. Performance evaluation of various officials varies depending on tasks, importance, values, difficulty of scoring. Thus, staff evaluation may be considered as a part of the staff management system and independent evaluation system to be applied within any company in a particular form. The article proposes a method of personnel assessment using a comprehensive assessment. It proposes the scale of evaluation, which is used to implement actions for measuring of the bonuses and personnel developing of the enterprise. A number of criteria for evaluating staff have been set. It distributes the share of each criterion in total amount, certain levels and their scores are also determined.
\end{abstract}

\section{Introduction}

The efficient evaluation system enables to develop, clarify and confirm selection criteria and, thus, to improve recruitment methods to be used. The results of periodic performance evaluation confirm (or do not confirm) the correctness of professional successfulness forecast made on the basis of selection methods and procedures used.

Interest in methods of the employee competence evaluation developed at the beginning of the previous century. The scientific approach to workplace management caused the occurrence of algorithms that assisted the companies to evaluate employees of various qualifications. Due to the analysis of efficiency, their professionalism has been defined and performance values have been calculated. Currently, professional skills of any employee may be insufficient to solve the current corporate purposes.

\section{Problem Statement}

As of today, one of the main criteria of efficient performance is staff evaluation. Staff evaluation ensures the receipt of comprehensive accurate information on the

\footnotetext{
* Corresponding author: agaverdieva@ukr.net
} 
employee. It enables to receive all required information on the certain employees and determine how one or another employee complies with the office requirements, as well as to improve the efficiency of staff management and development, and to improve psychological climate within the collective. Staff evaluation enables chief executives or staff management services to conduct more efficient human resources management, to improve and involve new employees instead of those who fail to perform their duties and, thus, to perform efficiently.

\section{Research Issues}

Staff evaluation priority refers to properly selected tools, staff evaluation methods, employee evaluation methodology, evaluation of personal and behavior features, demands in training, performance efficiency.

Staff evaluation is treated as part of management. Herewith, a method is required to study the qualitative composition of corporate staff, its advantages and disadvantages, and the basis for improving personal labor skills of the employee and further training [1]. As the evaluation results determine the employee position and their potential rotation, they are the important motivating factors for the labor improvement and attitude to the work.

\section{Purpose of the Study}

The purpose of the study is to conduct a comprehensive assessment of personnel and establish the criteria by which the assessment of personnel of the enterprise will be carried out.

Depending on purposes, according to scientists I.P. Migus and S.A. Chernenko, staff evaluation solves the following tasks: ensuring feedback between the employees and management; searching for measures to promote required specialists within the company; developing an efficient labor motivating system; obtaining psychological reference of required specialists; career planning and promotion; determining specialist competence level; determining the measures of satisfaction and interest of the employees in the company [2]. Thus, staff evaluation is the stable process of determining the compliance of qualitative staff features (skills, knowledge, motives) with the requirement of office or workplace [3, 4].

Staff evaluation provides for the comparison of certain human features professional and qualification level, business proficiency, performance with the certain parameters, requirements and standards. Thus, it can be stated that staff evaluation means the conclusion on the basis of comparison of "perfect" employee with that real human working for certain company and holding certain office. Staff evaluation refers to the determination to which extent each employee achieves any expected results and complies with the requirements derivative from his production tasks. Therefore, staff evaluation is the procedure to be conducted in order to determine the measures of compliance of personal employee features, quantitative and qualitative performance with standards, objectives to be achieved [5].

\section{Research Methods}

One of the basic difficulties which the company faces while evaluating the employees is the evaluation of managers. Often, it is due to interdependency of business and professional features of manager with the evaluation of final performance. Moreover, such evaluation takes a lot of time. Thus, it is reasonable to demonstrate the 
interdependency using the comprehensive evaluation. Parts of comprehensive staff evaluation are given in Figure 1.

It is known that the selection of evaluation system is subject to various factors: office, work experience, activity of subdivision and company in general, corporate culture existing within the company, as well as staff motivation.

Therefore, such problems exist: waste of time, imperfection of evaluation system and probability of personalism.

To avoid such situations, staff evaluation shall be conducted on an anonymous basis by independent experts so that the level of personalism is on the minimum level.

\begin{tabular}{|l|l|l|l|}
\hline $\begin{array}{l}\text { Evaluation of } \\
\text { nominee for a } \\
\text { vacant office }\end{array}$ & $\begin{array}{c}\text { Regular evaluation } \\
\text { (attestation) }\end{array}$ & $\begin{array}{l}\text { Evaluation of } \\
\text { business and } \\
\text { personal features }\end{array}$ & $\begin{array}{l}\text { Performance } \\
\text { evaluation }\end{array}$ \\
\hline $\begin{array}{l}\text { Data } \\
\text { collection } \\
\text { programs }\end{array}$ & $\begin{array}{l}\text { study of documents; } \\
\text { interviews; } \\
\text { questioning; } \\
\text { supervision }\end{array}$ & $\begin{array}{l}\text { labor } \\
\text { evaluation of } \\
\text { work difficulty }\end{array}$ \\
\hline cost
\end{tabular}

Fig. 1. Parts of comprehensive staff evaluation.

Quantitative figures may include as follows: standards established; performance of the best employees; plan of work performance. With regard to qualitative figures, they include as follows: knowledge, skills, abilities; employee's responsibility; employee's loyalty; ability to work in a team; leadership skills; ability to make decisions independently; independency in work execution.

With regard to heads and managers, there are qualitative figures to be evaluated as they indirectly influence over the results to be achieved; they should not be established by the number as they characterized the employee by criteria consistent with "perfect" beliefs how official duties and functions should be done, basic parts of such office. Thus, while evaluating the employees, it is reasonable to consider quantitative figures [6].

As of today, there are three requirements to the current manager: professional, business and personal skills. Relying upon basic requirements to managers, as well as reference and practical experience of leading national companies, there is the staff evaluation system for the company. Therefore, staff evaluation shall be conducted by business and professional skills.

Selection of evaluation criteria is based on reference in terms of specific features of corporate business. With regard to work experience, the employees may be divided into groups with the relevant level: work experience less than 3 years is evaluated as low level; $3-5$ years - average level; 5 - 10 - above the average; above 10 years - high level.

Educational level of the employees shall be evaluated as follows: high level - the employees with academic degree; above the average - the employees with higher education; average level - the employees with advanced education; low level refers to those employees with secondary education. Upon all other criteria, evaluation shall be conducted by the direct manager. Herewith, each feature of professional and business skills and performance shall have the own level to be scored as follows: low -0.5 ; average -1 ; above the average- 1.5 ; high -2 scores. 
Score from 0.5 to 2 shall be awarded to the employee for each feature in terms of specific value. Herewith, the ratio of professional level of the employee shall be established using a formula:

where $L$ - relevant level;

$$
R_{p r}=\sum L \times S V_{c}
$$

$S V_{c}$ - specific value of relevant criterion.

The employee shall comply with the requirements to be put against him upon official duties, context and work nature, and corporate requirements and corporate culture. The evaluation shall include not only potential abilities of the employees, professional level, their competence, but also the implementation of such possibilities in the process of the fulfillment of obligations assumed and achievement of corporate performance standards [7].

Through evaluation, criteria of staff evaluation shall be clearly distinguished. Herewith, for the employees the execution of work volume and products quality shall be of the great importance. Performance, but not personality shall be evaluated.

In table 1 there are the criteria of evaluation and specific value of corporate performance.

Table 1. Performance evaluation.

\begin{tabular}{|l|c|c|}
\hline \multirow{2}{*}{ Evaluation criteria } & \multicolumn{2}{|c|}{ Specific value } \\
\cline { 2 - 3 } & Employees & Managers \\
\hline Volume of work executed & 0.25 & 0.2 \\
\hline Quality of works executed & 0.25 & 0.3 \\
\hline Knowledge and compliance with safety procedure & 0.15 & 0.1 \\
\hline Compliance with technological process & 0.15 & 0.1 \\
\hline Timely work execution & 0.2 & 0.3 \\
\hline Total & 1 & 1 \\
\hline
\end{tabular}

Thus, evaluation of performance for the stated period shall be determined as the amount of evaluation of feature levels in terms of specific values. It shall mean the difficulty of work.

The comprehensive evaluation of the employees shall be calculated using the formula:

$$
C E=S h \times S_{S}+S h \times P,
$$

where, $S h$ - share of relevant skills per total evaluation, \%;

$S s$-scores of professional and business skills of the employee;

$P$-scores for performance.

The evaluation results are the basis for further decision of the certification commission and they are also focused on the evaluation of labor potential, career development, feedback and etc.

Moreover, the improved staff evaluation system, efficient evaluative technologies developed subject to corporate features, methodologies, unique skills and evaluation methods - all these in total may play a role of intellectual capital of the company. The staff evaluation system as the intellectual capital of the company occurs in such forms as: human capital - abilities, knowledge, skills, motivation of specialists in the field of staff evaluation. Therefore, we should say that the evaluation of labor activity of the employee as a part of total performance of the collective is quite difficult task. Those evaluation methods to be used as of today are quite subjective. The human evaluates the environment, including the behavior of other people, not through personal assessment, but through interpretation. Thus, the companies may use any evaluation method of individual performance. But the chosen method shall be simple and clear for the employees. Therefore, it should be noted that while evaluating performance the proper determination of quantitative and qualitative 
values reflecting final purposes of subdivision and company in general shall have practical importance.

The selection of performance evaluation criteria depends on affiliation with one or another category of the employees: heads, specialists, employees, workers.

The head performance is stated through progress of the company or branch or division: increase in profits, market share, increase in clients and etc., and through social and economic working conditions of the employees (for example, increase in salary, increase in discounts, etc.). The criteria of performance evaluation shall be subject to certain requirements. They shall: have direct and final influence with performance of the company; cause the achievement of corporate purposes; influence over at least $80 \%$ of performance of the employees. The criteria of performance evaluation shall be at least 5-6.

The selection of criteria shall be determined through the establishment of evaluation criteria, i.e. which tasks shall be solved through the used evaluation results. Thus, for example, if man purposes are the increase in performance and determination of salary amount, the evaluation shall be conducted upon the criteria of productivity. If the purpose is the promotion, there shall be criteria to determine the potential productivity at new workplace and etc [8]. The scheme of staff evaluation is given in Figure 2.

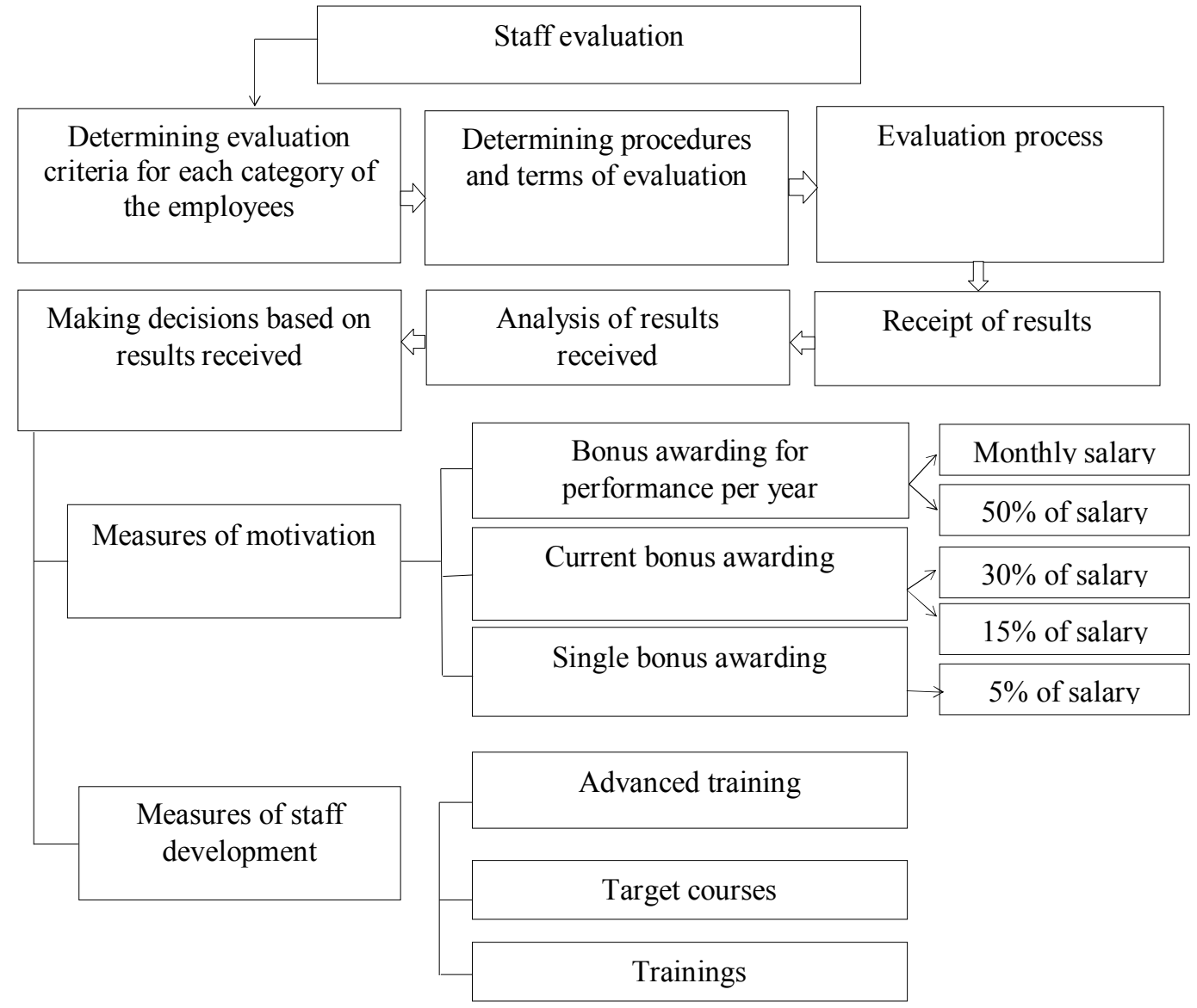

Fig. 2. Scheme of staff evaluation within the company.

The timing of evaluation depends on various factors: specific features of work, term of labor agreement, period of working cycle, significant change in productivity, work completion per each certain task and etc. 


\section{Findings}

Therefore, it is recommended to conduct the final evaluation of the heads and managers twice per year. Scores received in a current evaluation shall be summarized for certain periods and converted into average values. As a result, the decision on bonus awarding and staff training will be made.

The evaluation shall be conducted at the following scale:

$1.7-2$ - the employee holds the office. Such employee shall be included into reserve for the promotion and at the end of the year he will be paid with $100 \%$ monthly salary.

$1.4-1.6$ - the employee holds the office and at the end of the year he will be paid with $50 \%$ of one monthly salary. He may be in reserve for the promotion.

$1-1.3$ - the employee holds the office. He may be included into reserve provided he passes training or further training or education. Bonus award shall not be paid.

0.9 and less. The employee holds the office provided he passes further training. Then the employee shall have the repeated evaluation. In case of negative result, the employee shall be reduced. Bonus award shall not be paid.

The selection of evaluation method for the company studied is the unique task to be solved only by the head. Thus, the implementation of the staff evaluation system demonstrated in this paper work enables to justify all long-term and short-term corporate purposes.

\section{Conclusion}

Thus, it should be concluded that staff evaluation conduct within the company gives the possibility of: evaluating potential for the promotion and reduction of the risk of non-competent employee promotion; arrangement for feedback with the employees on quality of their performance; determining the necessity of staff training; determining costs for training; developing plans and programs for staff development; determining potential increase in the employee's qualification; considering potential changes in salary amount; making amendments to the staff motivation system.

\section{References:}

1. N.N. Kozak, Staff planning. Top-manager's library, 60 (2017)

2. I.P. Migus, S.A. Chernenko, Business inform, 10, 357-362 (2013).

3. L.V. Balabanova, Staff management: textbook, 512 (2006)

4. A.A. Vuchkovych-Stadnyk, Staff evaluation: clear algorithm of actions and quality practical solutions, 192 (2010)

5. O.A. Hrishnova, Human resources management: problems, theory and practice, 7 , 42-50 (2005)

6. Staff evaluation: [Electronic resource]. - Available at: https://hrliga.com/index.php? module=profession\&op=view\&id=1831

7. H.V. Nazarova. Growth and use of managerial staff - strategical approach: monographs, 216 (2012)

8. A.Ia. Kybanov, Corporate staff management: actual technologies of employment, adaptation and attestation: textbook, 360 (2016)

9. O.A. Havrysh, Staff management technologies: monographs, 528 (2017)

10. O. Krushelnitska, Staff management: Textbook, 304 (2005)

11. V.Ia. Chevhanova, Efficient economy, 4 (2014) 